Leading the Centers for Disease Control and Prevention (CDC), America's premier public health agency, is a challenging task at the best of times. Meet Julie Gerberding, head of the CDC in these difficult days of bioterrorism, and the first female director in the agency's 57-year-history.

It was during her residency at San Francisco General Hospital that Julie Gerberding became interested in public health. She treated people we now know to be the first AIDS patients, and working in that emerging epidemic drove home to her the devastating effect a disease can have on a population. She went on to earn a Master's degree in public health at the University of California, Berkeley, in 1990.

Gerberding also trained as a bench scientist when she completed a National Institutes of Health training fellowship in clinical pharmacology and infectious disease. "I am so glad I had that experience because it honed my scientific thinking and allows me to use that same scientific approach to public health problems," she says.

Those two distinct events were but a prologue to what will surely be the most important chapter in Gerberding's career. In July 2002, she was appointed director of the CDC. The announcement played heavily on her being the first woman to take on the role, and on her infectious disease knowledge. A factor unwritten in official press statements, but which might have contributed significantly to her appointment, was that she was selected particularly for her ability to manage the agency through a public health crisis, potentially the most critical that America has faced-the threat of bioterrorism.

Gerberding's predecessor, Jeffrey Koplan, had not handled the emergence of this threat-which began with the five deaths from anthrax-well. Critics charged that he was not visible enough to calm an alarmed public, and characterized the agency as ill-coordinated and unprepared to respond to a health crisis. Koplan resigned his post in March 2002. Gerberding, who joined the CDC in 1998 as director of the Division of Healthcare Quality Promotion, had proven to be a decisive leader during this time. That quality is now being tested as she leads the CDC in implementing a program of smallpox vaccination across the country.

The agency, Gerberding says, has a tradition of accommodating new health threats. "If you look back a few decades ago to when HIV infection was emerging,

\title{
Julie Gerberding
}

[the] CDC had to undergo a similar expansion to meet those challenges," she says. "We redirect investments to target the new problem, we work hard to get support through the budgetary process, we rapidly acquire, recruit or retrain the personnel needed to address it."

Redirecting large amounts of money, time and research toward combating diseases that may never arise is not wasteful, Gerberding says. "Emphasis on terrorism has created new challenges but also new

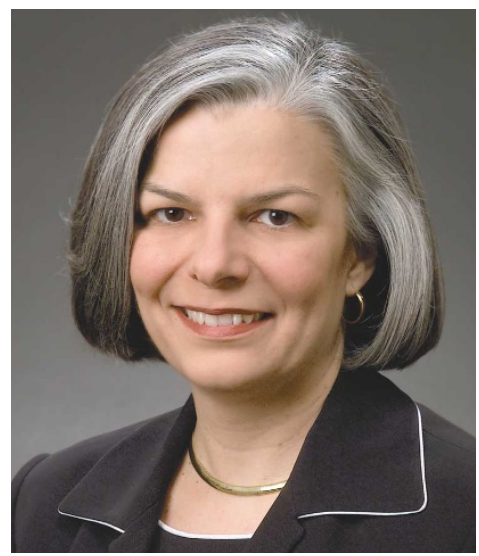

The First Lady of public health

opportunities. One of the most conspicuous of those opportunities is that the investments that are being made in terrorism containment and response really do strengthen our entire infrastructure and public health system," she says. "What we're doing to respond to bioterrorism is exactly the same set of capacities that will help us improve our ability to respond to emerging infectious diseases such as West Nile or pandemic influenza."

Public awareness of health issues is vital, Gerberding says, adding she has "worked very hard to expand the whole communications program at [the] CDC." Immediately after the anthrax attacks, she points out, the CDC asked various experts and the media to help the agency communicate with the public. To disseminate information about the smallpox vaccination program, for example, the agency has used public service announcements, held webcasts and satellite broadcasts to reach potential volunteers, and sent information kits to 3.5 million clinicians to describe the various medical aspects of the scheme.

Despite these efforts, the vaccination program has had a lukewarm response nationally (see page 249), with continuing concerns about how volunteers will be compensated for illness and how third parties will be protected from inadvertent inoculation by a vaccinee.

"When we planned the program we didn't anticipate that everyone would participate," Gerberding says of the low numbers. Some people choose not to be vaccinated because of contraindications, such as eczema or a compromised immune system, "and others, for whatever reason, have chosen not to be vaccinated," she says. The CDC's goal, she adds, is to have enough response capacity in a community so that in the case of a smallpox attack, the government would be prepared to implement vaccination clinics to protect the public. "We expected that there would be individuals who did not want to participate," she said. "Our unit of interest is the community."

Gerberding points out that President Bush has not as yet recommended vaccinating the general public. But "if we needed to do this in an emergency," she says, "we have more than enough doses of vaccine to protect every man, woman and child in America." The national pharmaceutical stockpile also has enough doses of vaccinia immune globulin (VIG), which is the first line of therapy for adverse reactions. Estimates suggest that, for every million people vaccinated, as many as 52 will suffer adverse effects and 2 will die. Ten million health care workers are expected to be immunized in the initial phase of vaccination. "We are in a rapid ramp-up of production of more VIG," she says.

The spotlight on bioterrorism aside, Gerberding points out that the CDC director has many other priorities. "I've just been presenting information on our efforts to combat cancer, and also preparing a talk on antimicrobial resistance, which is clearly an important public health program," she says. "On any given day our agency is fairly broadly focused on all the health priorities. Bioterrorism preparedness is certainly not the only priority on my desk."

Karen Birmingham, London 